\title{
Theoretical X-ray line profiles from colliding wind binaries
}

\author{
David B. Henley, Ian R. Stevens \\ School of Physics and Astronomy, The University of Birmingham, \\ Edgbaston, Birmingham B152TT, UK \\ Julian M. Pittard \\ Department of Physics and Astronomy, The University of Leeds, \\ Woodhouse Lane, Leeds LS2 9JT, UK
}

\begin{abstract}
We present theoretical X-ray line profiles calculated for a range of model colliding wind systems. In particular we have investigated how the line profiles vary with wind parameters. These profiles will be compared with high-resolution grating spectra of colliding wind binaries and will provide new insights into the structure of the X-ray emitting regions in such systems.
\end{abstract}

\section{Introduction}

X-ray observations of colliding wind binaries (CWBs) enable us to probe the wind-wind interaction zone and to determine gross wind parameters such as mass-loss rates, wind velocities and chemical abundances. However, the abilities of Chandra and XMM-Newton to resolve line shifts down to a few hundred $\mathrm{km} \mathrm{s}^{-1}$ offer us the potential to study in detail the distribution and dynamics of the hot, X-ray emitting plasma in stellar winds. Single O-type stars are predicted to produce blue-shifted emission lines (Ignace 2001; Owocki \& Cohen 2001), as has been observed in the Chandra grating spectrum of the O4I(n)f star $\zeta$ Pup (Cassinelli et al. 2001). However, until now a detailed investigation of X-ray line profiles from CWBs has not been made.

\section{Details of the line profile calculations}

Our line profile calculations are based upon 2D hydrodynamical simulations of model colliding wind systems. The systems are characterized by the wind momentum ratio $R$, defined by

$$
R=\frac{\dot{M}_{\mathrm{A}} v_{\mathrm{A}}}{\dot{M}_{\mathrm{B}} v_{\mathrm{B}}}
$$

where $\dot{M}_{\mathrm{A}, \mathrm{B}}$ are the mass-loss rates and $v_{\mathrm{A}, \mathrm{B}}$ are the wind velocities of the two stars (A is the star with the more powerful wind).

Each cell on the grid is assumed to produce a thermally broadened profile whose centre is shifted according to the line-of-sight velocity of the gas in that 

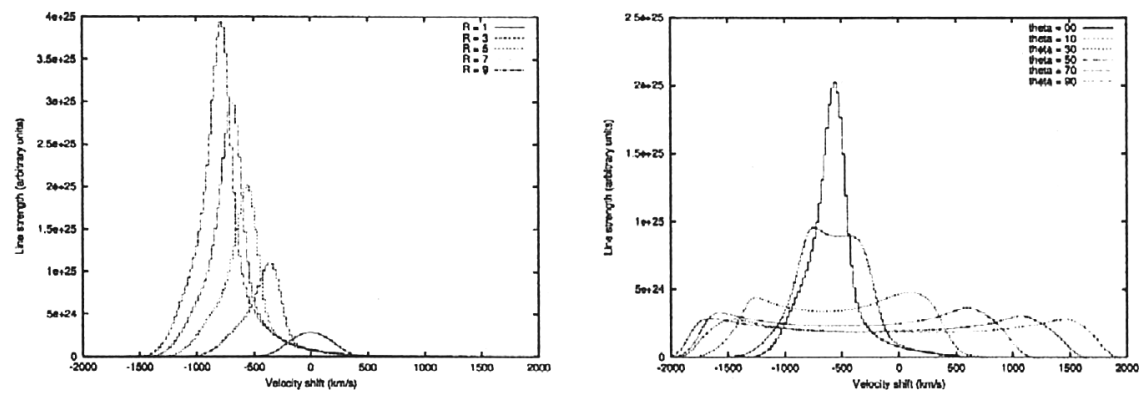

Figure 1. $\operatorname{Mg}$ XII Ly $\alpha$ line profiles from model colliding wind binaries. The left hand plot shows how the profile varies with $R$ when the system is viewed along the line of centres from behind the secondary. The right-hand plot shows how the profile varies with viewing angle for $R=5\left(\theta=0^{\circ}\right.$ corresponds to viewing the system along the line of centres from behind the secondary).

cell. The line profile is calculated by summing the contributions from the whole grid.

\section{Results}

We have calculated line profiles for the Ly $\alpha$ lines from $\mathrm{O}, \mathrm{Ne}, \mathrm{Mg}, \mathrm{Si}, \mathrm{S}$ and $\mathrm{Fe}$. Typical results are shown in Figure 1. When the system is viewed along the line of centres the lines become more blueshifted as $R$ increases because the windwind interaction zone is bent more towards the observer. When the system is viewed at an angle to the line of centres the profiles become broader and flatter.

The line blueshifts indicate that the Fe line is produced in the very hot gas near the line of centres, with the other lines originating progressively further out (the $\mathrm{O}$ line forming furthest from the line of centres). The FWHMs are larger than the expected thermal widths, indicating that the profiles are not simply dominated by thermal broadening.

\section{Prospects}

Preliminary photon-counting simulations based on our theoretical line profiles indicate that significant line shifts and line widths larger than the expected thermal widths are detectable by Chandra and XMM-Newton. By comparing our results with high-resolution grating spectra of CWBs we shall increase our understanding of the X-ray emitting region in these interesting systems.

\section{References}

Cassinelli, J.P., Miller, N.A., Waldron, W.L., MacFarlane, J.J., Cohen, D.H. 2001, ApJ (Letters) 554, L55

Ignace, R. 2001, ApJ (Letters) 549, L119

Owocki, S.P., Cohen, D.H. 2001, ApJ 559, 1108 